\title{
Analysis of motion of the three wheeled mobile platform
}

\author{
Anna Jaskot ${ }^{1}$, Bogdan Posiadała ${ }^{1, *}$ \\ ${ }^{1}$ Institute of Mechanics and Machine Design Fundamentals, Czestochowa University of Technology, \\ ul. Dąbrowskiego 73, 42-201, Częstochowa, Poland
}

\begin{abstract}
The work is dedicated to the designing motion of the three wheeled mobile platform under the unsteady conditions. In this paper the results of the analysis based on the dynamics model of the three wheeled mobile robot, with two rear wheels and one front wheel has been included The prototype has been developed by the author's construction assumptions that is useful to realize the motion of the platform in a various configurations of wheel drives, including control of the active forces and the direction of their settings while driving. Friction forces, in longitudinal and in the transverse directions, are considered in the proposed model. Relation between friction and active forces are also included. The motion parameters of the mobile platform has been determined by adopting classical approach of mechanics. The formulated initial problem of platform motion has been solved numerically using the Runge-Kutta method of the fourth order. Results of motion analysis with motion parameters values are determined and sample results are presented.
\end{abstract}

\section{Introduction}

Mobile platforms are designed as nonholonomic dynamical systems with its wheels assumed to be rolling without slipping or skidding. Research of rolling without slippage of both the kinematics and dynamics of motion have been performed and the results have been presented among others in [1]. Thus, in order to properly mapped the motion of the wheeled mobile platform the slippage need to be taken into account. The farm vehicles designed to accomplish agricultural tasks in order to detect sliding which is different from the one occurring in cars have been considered in [2]. The internal dynamics of the vehicle with one front steering wheel has been described in [3]. In this work the model and some sample results of motion simulations of the mobile platform with three steering wheels are presented. Approach of considering the slip during motion and the model of single wheel dynamics for three-wheeled mobile robot with friction coefficient as a function of wheel slip have been discussed in [4]. The three kinematical aspects of mobile robots, such as mobility, control, and positioning, have been detailed reviewed in [5]. The dynamical model is important. The control strategy with kinematics of the system and with the

\footnotetext{
* Corresponding author: bogdan.p@imipkm.pcz.pl

Reviewers: Marek Macko, Ján Vavro Jr.
} 
velocity controller based on the robust sliding mode control technique have been discussed and developed in [6].

The novel method of tracking the reference trajectory and linearizing the nonlinear behaviour of robot under the influence of uncertainty and disturbance by using the hybrid controller has been introduced in [7]. In the same paper authors have presented the kinematic and dynamics models, and the results have been presented by using Langrange formulation.

In this work, besides the model of dynamics of motion of the mobile platform with three wheel drive, the simulation results have been included. In the model the linear velocity and acceleration as well as the angular velocity and acceleration of the center of mass, and the displacement of the center of mass of the platform have been performed by using numerical method. The aim of this research was to present an analysis of the behaviour of platform's motion.

The analysis of motion of the three wheeled mobile platform has been conducted. The conclusions have been formulated on the basis of comparison of the two cases of motion. First case concerns motion without skidding or slipping and the second when platform have been working under both mentioned: slipping and skidding. This comparison is useful in correct understanding and violating the method, presented in this work, and its results. The model of dynamics of motion has been formulated by adopting classical approach of the mechanics. The idea to design the three wheeled robot itself isn't new, but to design three wheeled mobile platform with three steering wheels equipped in three drive units is an author's model. By using such a system the analysis of motion in unsteady conditions have been accomplished.

In the next section the model of the platform has been introduced. In the third section the theoretical and mathematical models have been presented. In the fifth section the simulation results with analysis of the graphical representations have been included. The conclusions and vision of further research have been gathered and described in the last section of this paper.

\section{Model of the three wheeled mobile platform}

The model has been made on the basis of the prototype model of the four wheeled mobile platform described in [8]. A modular design is useful in analysing every possible position of the platform and its wheels. It was assumed, that each drive wheel will be steering wheel (possibility of rotate around the $Z$-axis), and the drive units could be subjected to different values of drive torques resulting from the construction and ability of the adopted electric motors.

Model of the analysed system, which is the mobile platform with three wheel drive has been presented in Fig. 1. The model of dynamics has been determined in the reference frame $O X Y Z$. Point $S$ has been denoted as a center of mass of the platform. The Sxyz coordinates represent the local coordinate system of the center of mass. Rotation angle between the $X$ and $x$ axes (and the same between the $Y$ and $y$ axes) has been denoted as $\beta$. It has been assumed that the platform will work on a flat surface (permissible inclination angle should not exceed $5^{\circ}$ ). The motion has been analyzed as a planar motion, and, since during motion has not occurred any changes between parts of the system, the platform has been considered as a rigid body. Each of the three steering wheels have been considered in order to determine forces occurring during platform work. 


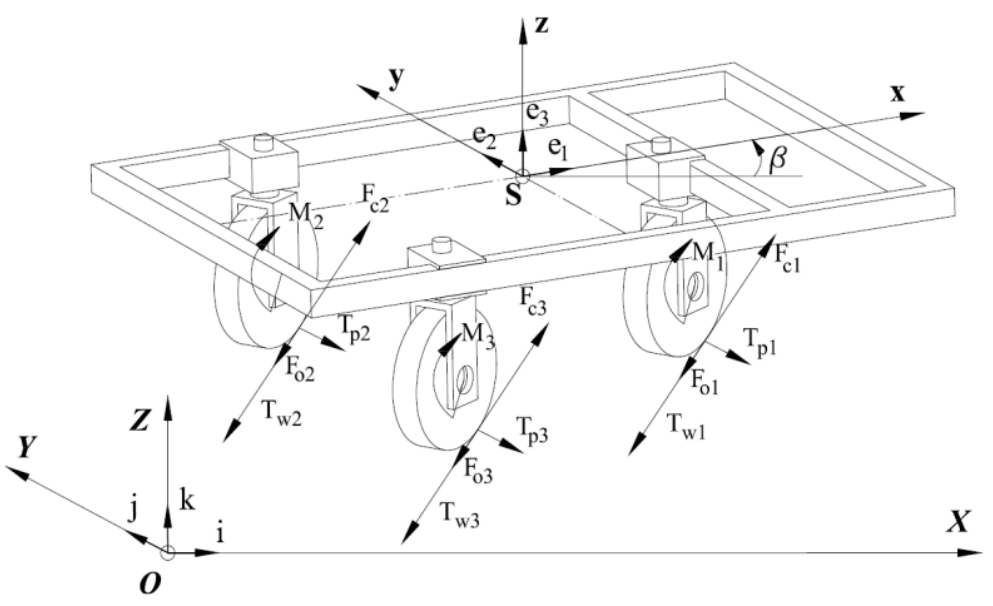

Fig. 1. The prototype model of the mobile platform in the reference frame

The $O_{\mathrm{i}} x_{\mathrm{i}} y_{\mathrm{i}} z_{\mathrm{i}}$ (Fig. 2) system is connected to every single wheel. The forces characterizing the dynamics of motion are schematically presented in Fig. 2. Based on the total mass of the platform calculated to a single wheel, the $N_{\mathrm{i}}$ force represents a reaction from the ground. The $M_{\mathrm{i}}$ is a drive torque and it is the initial data in this research. From the drive torque comes the active force $\mathbf{F}_{\mathrm{i}}$. The friction forces have been designated as $\mathbf{T}_{\mathrm{wi}}$ in longitudinal and $\mathbf{T}_{\mathrm{pi}}$ in transverse directions. The $\mathbf{F}_{\mathrm{oi}}$ force represents the resistance force, which prevented from starting movement of the platform and decelerated when the drive torque has been switched off.

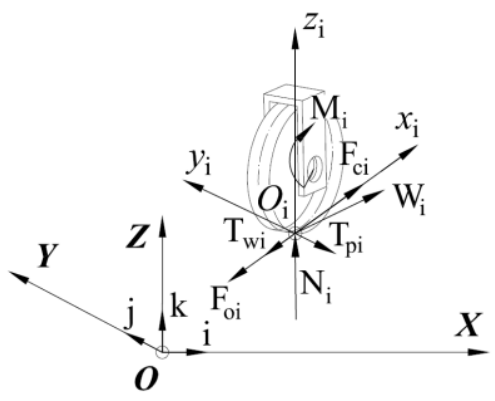

Fig. 2. Forces of the i-th wheel of the platform

\section{Model of dynamics of motion}

In this section the theoretical and mathematical models have been included. Model of dynamics of motion of the wheeled robots has been determined when considering the system as a rigid structure and each of the three wheels and the steering assembly. To complete obtaining the mathematical model of the dynamics of the mobile platform it is a very important to look into the dependencies between the ground, wheels and the system with taking into account all forces applied upon the mobile platform system. Based on the initial value of the drive torque the active force Fci can be determined from the Eq. 1 .

$$
\mathbf{F}_{\mathrm{ci}}=\frac{M_{\mathrm{i}}}{r} \cdot \mathbf{e}_{1}^{i}
$$

where: $r$ - radius of an $i$-th drive wheel. 
By adopting the Coulomb friction model the friction forces in both longitudinal and the transverse directions can be obtained from the formulas in Eqs. 2 and 3, where the $\mu_{\mathrm{w}}$, and $\mu_{\mathrm{p}}$ are the coefficients of friction (in the longitudinal and transverse direction), and the $v_{\mathrm{wi}}$, and $v_{\mathrm{pi}}$ are the velocity components (in the longitudinal and transverse direction, as well).

$$
\begin{aligned}
& \mathbf{T}_{\mathrm{wi}}=-\mu_{\mathrm{w}} \cdot N_{\mathrm{i}} \cdot \operatorname{sign}\left(v_{\mathrm{wi}}\right) \cdot \mathbf{e}_{1}^{i} \\
& \mathbf{T}_{\mathrm{pi}}=-\mu_{\mathrm{p}} \cdot N_{\mathrm{i}} \cdot \operatorname{sign}\left(v_{\mathrm{pi}}\right) \cdot \mathbf{e}_{2}^{i}
\end{aligned}
$$

where $N_{\mathrm{i}}$ is a reaction deriving from the wheel load on the ground.

The dependence between the active and passive forces cause the slippage or lack thereof. When the friction forces overcome the active forces, the platforms wheel will slip on the ground, otherwise - it won't. In this work both possibilities have been considered.

The motion description can be explained as the superposition of two motions compound of translation and rotation. The translational motion is when all wheels slide in the same direction (a lateral or side motion). The rotational motion is when the wheels does not slide exactly in the same way, this generates move around center of mass. The equation of a translational motion (the progressive motion) equation is presented in Eq. 4, and the rotational motion around $\mathrm{Z}$-axis is presented in Eq. 5 .

$$
\begin{gathered}
m \mathbf{a}=\sum_{i=1}^{3} \mathbf{W}_{\mathrm{i}} \\
\frac{d \mathbf{K}}{d t}=\sum_{i=1}^{3} \mathbf{s}_{\mathrm{i}} \times \mathbf{W}_{\mathrm{i}}+\sum_{i=1}^{3} \mathbf{M}_{\mathrm{mi}}
\end{gathered}
$$

where: $m$ is the total mass of the platform (with an equipment), $\mathbf{a}$ is the acceleration of the center of mass of the platform, $\mathbf{W}_{\mathrm{i}}$ is the resultant force containing the described active and passive forces, $\mathbf{K}$ is the angular momentum vector of the whole platform, $\mathbf{s}_{\mathrm{i}}$ is the location vectors of each of the drive wheels, $\mathbf{M}_{\mathrm{mi}}$ is moment deriving from the rotational motion around center of mass during the slippage.

After neglecting the $\mathbf{M}_{\mathrm{mi}}$, to obtain the motion parameters the transformation the above equations in form of the differential equations have been made Eqs. 6-8.

$$
\begin{gathered}
\ddot{X}=\frac{1}{m} \sum_{i=1}^{3} W_{\mathrm{ix}} \\
\ddot{Y}=\frac{1}{m} \sum_{i=1}^{3} W_{\mathrm{iy}} \\
\ddot{\beta}=\frac{1}{I_{\mathrm{z}}} \sum_{i=1}^{3}\left(s_{\mathrm{ix}} \cdot W_{\mathrm{iy}}-s_{\mathrm{iy}} \cdot W_{\mathrm{ix}}\right)
\end{gathered}
$$

where: $\ddot{X}$ is designated as the acceleration of center of mass on the $X$-axis in reference frame, $\ddot{Y}$ is the acceleration of center of mass on the $Y$-axis in reference frame, $\beta$ is an angular acceleration around center of mass of the platform.

By using the Runge-Kutta method of the fourth order, the motion parameters have been determined and the sample results are presented. 


\section{Sample results of motion simulation}

This chapter has been dedicated to the solution results of the dynamics of motion of the platform with three wheel drive. The results including positioning of the platforms wheel with its trajectories of motion, velocities and accelerations in two cases. First case, when none of the platform's wheels has slipped, and the second - when platform has been under slippage conditions. The second option has been made as a result of modification the friction coefficient in the second wheel and representing situation with moved center of mass of the platform from the central position. The size of the platform is equal one meter along the $X$ axis and $0.7 \mathrm{~m}$ along the $Y$ axis. The front wheel has been placed in local coordinates $x=0.4 \mathrm{~m}$ and $y=0$. The two back wheels have been situated in $x=-0.2 \mathrm{~m}$ (both), and the $y=0.35 \mathrm{~m}$ (second wheel) and $y=-0.35 \mathrm{~m}$ (third wheel). Initially it was assumed, that by placed the load in the center of the platform will be convenient, because the weight distribution will be similar. Modification of placement load in different way will bring the new position of the center of mass of the platform, and those forces will be different.

The initial parameters, assumed on the basis of the engines from the catalogue [9] and of prototype design, have been gathered in the Tab. 1 .

Table 1. Initial parameters in the first case

\begin{tabular}{|l|c|c|}
\hline Initial values & Symbol & Value \\
\hline Acceleration due to gravity $\left[\mathrm{m} / \mathrm{s}^{2}\right]$ & $\mathrm{g}$ & 9.81 \\
\hline Radius of wheel $[\mathrm{m}]$ & $\mathrm{r}$ & 0.2 \\
\hline Total worktime $[\mathrm{s}]$ & $\mathrm{t}_{\mathrm{c}}$ & 20 \\
\hline Drive torque $[\mathrm{Nm}]$ & $\mathrm{M}_{\mathrm{i}}$ & 15 \\
\hline Coefficient of friction for the longitudinal direction & $\mu_{\mathrm{w}}$ & 0.2 \\
\hline Coefficient of friction for the transverse direction & $\mu_{\mathrm{p}}$ & 0.1 \\
\hline Mass of the platform $[\mathrm{kg}]$ & $\mathrm{m}$ & 100 \\
\hline Starting position of the center of mass of the platform $[\mathrm{m}]$ & $\mathrm{S}$ & $\mathrm{X}=0.0, \mathrm{Y}=0.0$ \\
\hline Angle of inclination the platform $[\mathrm{rad}]$ & $\mathbf{b}_{\mathrm{p}}$ & $\mathrm{Pi} / 6(30 \mathrm{deg})$ \\
\hline
\end{tabular}

The value of the drive torque means the maximum value of the drive torque, which is given in a trapezoidal function in Fig. 3.

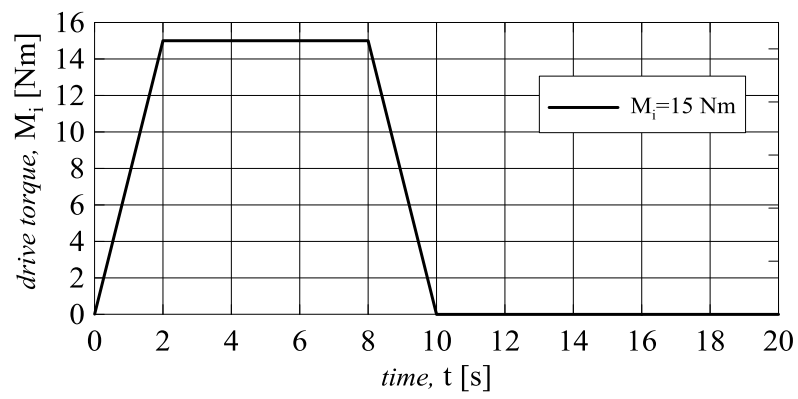

Fig. 3. Drive torque representation

From the initial parameter the active forces have been obtained. The passive forces, based on the total mass of the platform (constructional and external load) and the friction coefficients equal in every wheel, have been calculated. A graphical comparison of the relation between active and friction forces in every wheel has been shown in Fig. 4. 


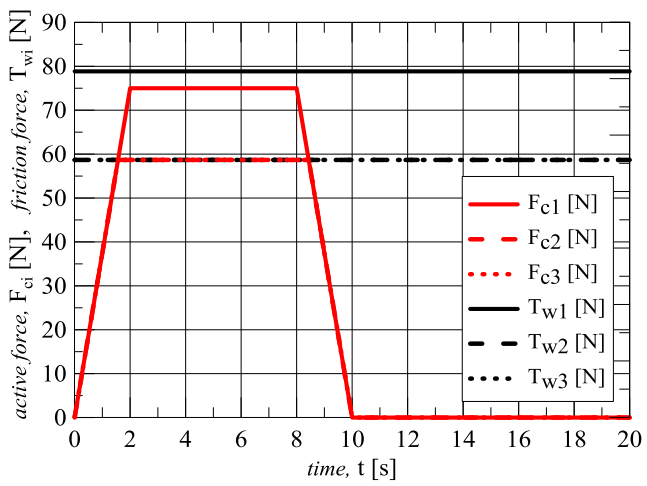

Fig. 4. Relation between active and friction forces during motion

The trajectory of motion of the center of mass of the platform without slippage has been presented in Fig. $6 \mathrm{~b}$. The representation of motion parameters in translational and rotational motions in this case are presented in Fig. 5a-b and $6 a$.

a)

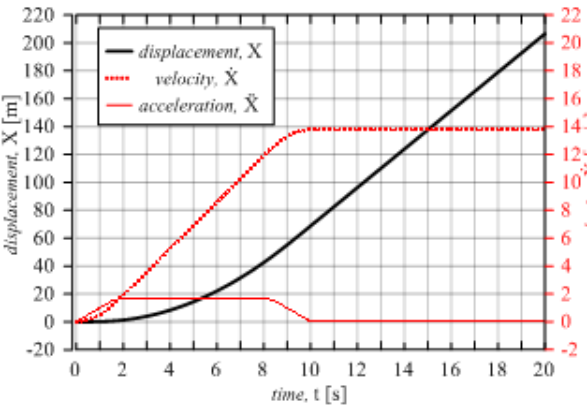

b)

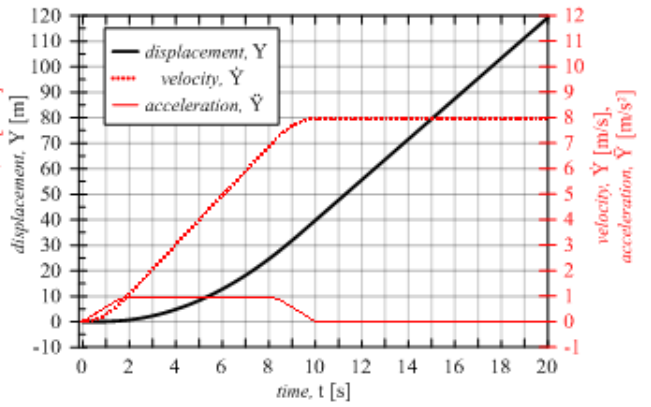

Fig. 5. Motion parameters a) in $\mathrm{X}$-axis b) in $\mathrm{Y}$-axis in the reference frame in translational motion

a)

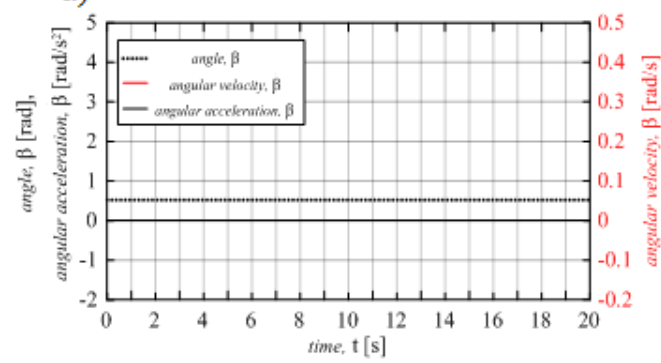

b)

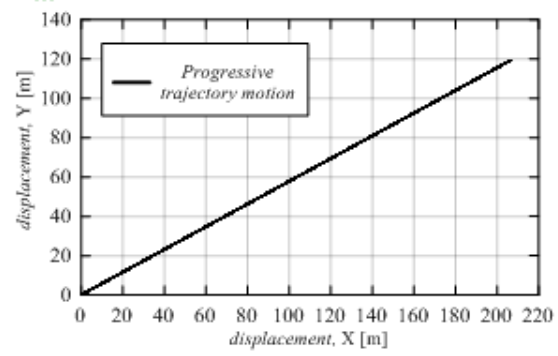

Fig. 6. a) Motion parameters in rotational motion, b) trajectory of motion of the center of mass

The second case of this analysis has been made on the basis of the description from the beginning of this section. The initial parameters in this case have been gathered and presented in Tab 2. The coefficients of friction have been reduced by a half of their initial values. This can represent an unpredictable situation on the surface at which the platform could work. The center of mass, as a result of different load localization on the platform, have been changed along the $\mathrm{Y}$-axis at half distance. 
Table 2. Initial parameters in the second case

\begin{tabular}{|l|c|c|}
\hline Initial values & Symbol & Value \\
\hline Acceleration due to gravity $\left[\mathrm{m} / \mathrm{s}^{2}\right]$ & $\mathrm{g}$ & 9.81 \\
\hline Radius of wheel $[\mathrm{m}]$ & $\mathrm{r}$ & 0.2 \\
\hline Total worktime $[\mathrm{s}]$ & $\mathrm{t}_{\mathrm{c}}$ & 20 \\
\hline Drive torque $[\mathrm{Nm}]$ & $\mathrm{M}_{\mathrm{i}}$ & 15 \\
\hline Coeff. of friction in longitudinal direction (wheels: 1,3$)$ & $\mu_{\mathrm{w}}$ & 0.2 \\
\hline Coeff. of friction in transverse direction (wheels: 1,3$)$ & $\mu_{\mathrm{p}}$ & 0.1 \\
\hline Coeff. of friction in longitudinal direction (wheel: 2$)$ & $\mu_{\mathrm{w}}$ & 0.1 \\
\hline Coeff. of friction in transverse direction (wheel: 2$)$ & $\mu_{\mathrm{p}}$ & 0.05 \\
\hline Mass of the platform $[\mathrm{kg}]$ & $\mathrm{m}$ & 100 \\
\hline Starting position of the center of mass of the platform $[\mathrm{m}]$ & $\mathrm{S}$ & $\mathrm{X}=0, \mathrm{Y}=0.175 \mathrm{~m}$ \\
\hline Angle of inclination the platform [rad] & $\mathbf{b}_{\mathrm{p}}$ & $\mathrm{Pi} / 6(30 \mathrm{deg})$ \\
\hline
\end{tabular}

In this case the same course of the drive torque from Fig. 3 have been used. Because of modification of the coefficients of friction the passive forces have changed and the relation between active and friction forces has been presented in Fig. 7.

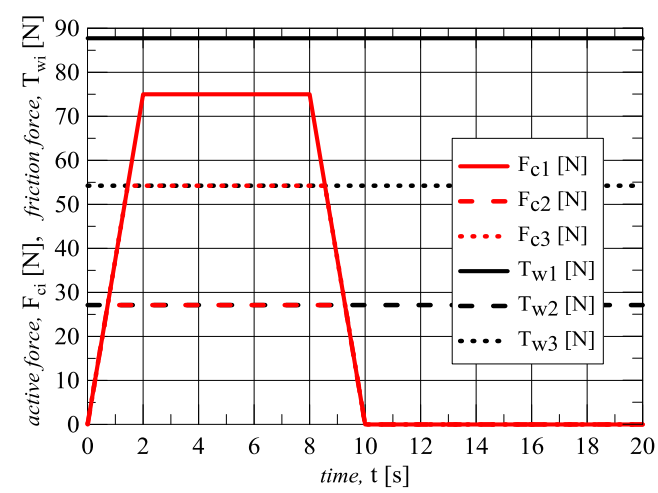

Fig. 7. Relation between active and friction forces during motion

The motion parameters have been presented in Fig. 8a-b. Those concerns progressive motion and have been shown in $\mathrm{X}$ and $\mathrm{Y}$ axes of the reference frame.

a)

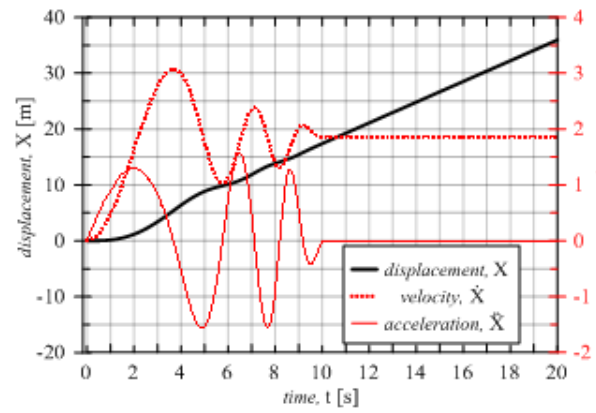

b)

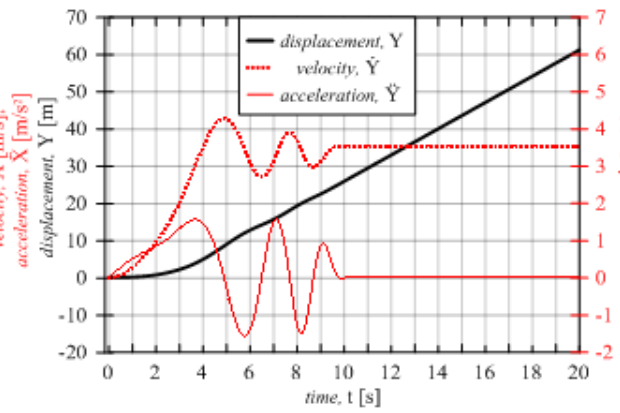

Fig. 8. Motion parameters a) in $\mathrm{X}$-axis b) in $\mathrm{Y}$-axis in the reference frame in translational motion in the second analyzed case 
The results deriving from the slippage and concerning the rotational motion of the platform have been presented in Fig. 9. As it is presented, the velocities after turning off the drive torque haven't changed. This is a result of assumption, that the resistance forces were equal zero.

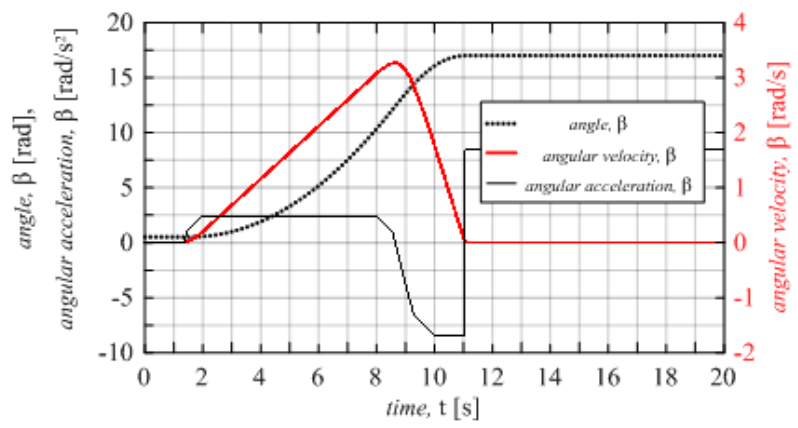

Fig. 9. Motion parameters in rotational motion when the slippage occurred

The trajectory of the superposition of the two motions is presented in Fig. 10.

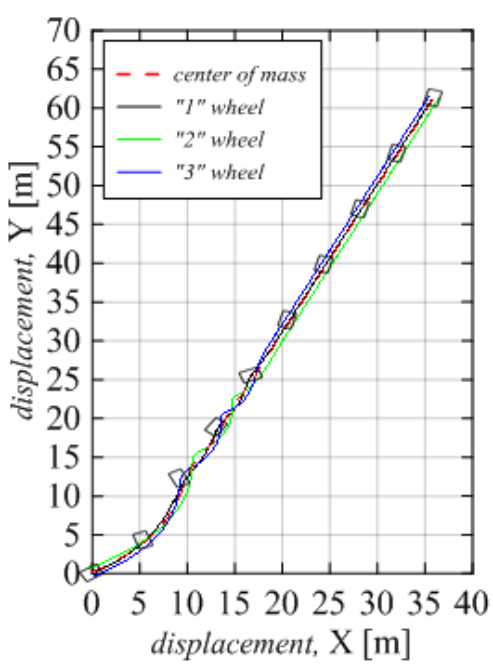

Fig. 10. Trajectory of motion of the center of mass in progressive motion and rotational motion around the $\mathrm{Z}$-axis

The platforms rotations have been presented by the rectangles attached to the trajectory lines in the figure.

\section{Conclusions}

The analysis of motion under and without slipping has been performed. The model of dynamics has been determined and the sample results of platform motion analysis have been presented. The model is useful in examining wheeled vehicles designed to work under unsteady conditions. The solution presented in the paper is a base of further studies of the dynamics of motion with taking into account the slippage conditions. Presented in the paper the mileages of changes the friction forces can cause slippage with respect to the wheels, where the determined values of friction forces reach its limit values (exceed the developed friction). 
The solution presented in the work allows to study the behaviour of the platform also while slippage and in the circumstances to refrain the platform from falling into the skid. Direction of further studies concerns the solution of refraining to fall into the skid.

\section{References}

1. A. Jaskot, B. Posiadała, S. Śpiewak, Dynamics model of the mobile platform for its various configurations. XXI International Polish-Slovak Scientific Conference Machine Modeling and Simulations, Hucisko, Poland (06-08 september 2016), Proceedia Engineering 177, 162-167 (2016)

2. R. Lenain, B. Thuilot, C. Cariou, P. Marinet, High accuracy path tracking for vehicles in presence of sliding: Application to farm vehicle automatic guidance for agricultural tasks. Autonomous Robotics 21, 79-97 (2006)

3. M. Eghtesad \& D. S. Necsulescu, Study of the internal dynamics of an autonomous mobile robot. Robotics and Autonomous Systems 54, 342-349 (2006)

4. R. Balakrishna, A. Ghosal, Modeling of slip for wheeled mobile robots. IEEE Transactions on Robotics and Automation 11 (1), 126-132 (1995)

5. N. Sidek, N. Sarkar, Dynamic Modeling and Control of Nonholonomic Wheeled Mobile Robot when subjected to wheel slip. (VDM Verlag Dr. Müller Aktiengesellschaft \& Co. KG, Saarbrücken, 2009)

6. A. El-Sayed, I. Bayoumi, Wheeled mobile robot trajectory tracking using sliding mode control. Journal of Computer Science, 48-55 (2016)

7. Z. A. Ali, D. Wang, M. Safwan, W. Jiang, M. Shafiq, Trajectory Tracking of a Nonholonomic Wheeled Mobile Robot Using Hybrid Controller. International Journal of Modeling and Optimization 6 (3), 136-141 (2016)

8. A. Jaskot, B. Posiadała, S. Śpiewak, Motion modeling of the four-wheeled mobile platform under slippage conditions. Machine Dynamics Research 40 (2), 43-53 (2016)

9. http://pl.kwapil.com/downloads/maxon-ec-motor.pdf (24 july 2017) 